\title{
Propuesta de Tareas para el Desarrollo del Pensamiento Variacional en Estudiantes de Ingeniería
}

\author{
Ana M. Báez ${ }^{(1) \star}$, Yoan Martínez-López ${ }^{(2)}$, Olga L. Pérez ${ }^{(2)}$ y Roger Pérez ${ }^{(2)}$ \\ (1) Universidad ISA, Departamento de Matemática, Avenida Presidente Antonio Guzmán Fernández, Km \\ 51/2, la Herradura, Santiago, República Dominicana (e-mail: ana26691@gmail.com) \\ (2) Universidad de Camagüey Ignacio Agramonte Loynaz, Grupo de Investigaciones de Matemática \\ Educativa, Circunvalación Norte, Km. 51/2, CP. 70300 Camagüey, Cuba. \\ (e-mail: yoan.martinez@reduc.edu.cu, olga.perez@reduc.edu.cu, roger.perez@reduc.edu.cu)
}

${ }^{*}$ Autor a quien debe ser dirigida la correspondencia.

Recibido Sep. 26, 2016; Aceptado Nov. 17, 2016; Versión final Ene. 17, 2017, Publicado Jun. 2017

\begin{abstract}
Resumen
En este artículo se describe una propuesta de tareas para desarrollar el pensamiento variacional en los estudiantes de ingeniería. Las tareas tienen como referentes teóricos a los procesos de tratamiento y conversión de los registros de representación semiótica, los procesos de coordinación reflexiva para la encapsulación y des-encapsulación del proceso-objeto, la comprensión de los conceptos matemáticos a partir de su funcionalidad, y la re-significación de los saberes matemáticos. Se precisaron los niveles del desempeño (reconocimiento, relación y deducción formal) de los estudiantes en la solución de ejercicios matemáticos en los cuales deben emplear el pensamiento variacional. Se desarrolló un experimento con dos grupos de estudiantes de ingeniería y con el mismo profesor. En el grupo experimental se implementaron las tareas propuestas, y en el grupo control se trabajó de forma tradicional. Los resultados mostraron que de los tres niveles de desempeños analizados, los niveles de relación y de deducción formal fueron superiores en el grupo experimental y que existían diferencias significativas entre las calificaciones de los estudiantes que conforman los dos grupos.
\end{abstract}

\section{Tasks Proposal for the Development of Variational Thinking in Engineering Students}

\begin{abstract}
This paper describes a proposal of tasks to develop variational thinking in engineering students. The tasks have as theoretical references the processes of treatment and conversion of semiotic representation registers, processes of reflexive coordination for the encapsulation and des-encapsulation of the objectprocess, the understanding of mathematical concepts based on their functionality, and the new meaning of mathematical knowledge. The levels of performance (recognition, relationship and formal deduction) of the students in the solution of mathematical exercises in which they must use the variational thought were defined. An experiment was developed with two groups of engineering students and with the same professor. In the experimental group, the proposed tasks were implemented, and the control group worked in a traditional form. The results showed that of the three performance levels analyzed, levels of relation and formal deduction were higher in the experimental group and that there were significant differences between the scores of the students that formed the two groups.
\end{abstract}




\section{INTRODUCCIÓN}

El descubrimiento del cálculo es uno de los grandes logros intelectuales de la civilización y ha sido utilizado por más de tres siglos como herramienta cuantitativa para la investigación de problemas científicos. Es esencial para áreas de la matemática (probabilidad, topología, teoría de grupos y álgebra, geometría, teoría de números) y otras áreas como la tecnología moderna, la física, la medicina, la mecánica, etc. (Kleiner, 2001). La misión fundamental de su enseñanza, en la educación superior, es formar profesionales que se apropien de los conceptos matemáticos y que logren aplicarlos en otros contextos diferentes al cual se aprendieron.(De Las Fuentes, Arcos y Navarro, 2010), de forma tal que sean capaces de enfrentar y resolver, con independencia y creatividad, los problemas que se les presenten en la sociedad, mediante la interpretación y aplicación de conceptos y métodos matemáticos, y adaptarse con relativa facilidad a un mundo cambiante. Investigadores nacionales y extranjeros en el campo de la Educación Matemática se han circunscrito a problemáticas relacionadas con el estudio del pensamiento matemático, en las que se considera que su desarrollo posibilita al estudiante describir, organizar, deducir consecuencias, apreciar patrones y regularidades, argumentar, usar diferentes sistemas de representaciones, interpretar y relacionarse con determinadas situaciones que posibilitan matematizar la realidad (Cuevas, 2012; Hannula, 2013; Almeida y Palharini, 2012).

Una de las problemáticas estudiadas es la relacionada con el pensamiento variacional, la cual tiene tres aristas fundamentales (Cantoral, 2004) que son asumidas en la investigación, y se desarrollan a partir de la problematización del saber matemático, ellas son: i) El estudio de las estructuras variacionales desde el punto de vista matemático y desde lo fenomenológico; ii) El estudio de los saberes matemáticos que los estudiantes ponen en juego al estudiar los conocimientos relacionados con la variación y el cambio; iii) Los problemas sociales que se estudian matemáticamente, a través de situaciones variacionales, en diferentes contextos educativos. Su estudio representa una vía para atender algunas de las dificultades que los estudiantes presentan en la adquisición de diversos patrones, en el establecimiento de conexiones entre aquellos conceptos de fuertes componentes dinámicos, sus representaciones gráficas y el movimiento real de los objetos en sus contextos (Cantoral y Farfán, 1998; Ruiz, 2009; Ordoñez y Alonso, 2013; Cabeza y Mendoza, 2016).

En este sentido, investigadores como Cantoral y Farfán, (1998), Arrieta y Díaz, (2015) y Font, Godino y Gallardo, (2013), entre otros, reportan la existencia de limitaciones en los estudiantes y profesores, al tratar situaciones que exigen algún tipo de estrategia variacional, como es el análisis del comportamiento y variación de gráficas; identificación de las cantidades que intervienen en una situación, cuáles cambian y cuáles permanecen fijas; determinación de las relaciones de dependencia entre dos variables; establecimiento de la relación entre razón de cambio y cociente incremental, y conversión del registro verbal al registro gráfico.

Estas limitaciones influyen negativamente en las expectativas sobre los contextos de aprendizaje que les gustaría a los estudiantes tener en los estudios universitarios, los cuales aspiran a tener experiencias de aprendizaje en que puedan valorar la funcionalidad de los conocimientos en contextos diversos (Paoloni, Martín y Chiecher, 2016), y esto está dado, fundamentalmente, porque no se aprovechan adecuadamente las potencialidades del contenido matemático en función del desarrollo del pensamiento variacional, lo que está afectando el desempeño de los estudiantes en la solución de ejercicios matemáticos donde tengan que emplearlo. En este contexto, el objetivo de la investigación estuvo orientado a mejorar el desempeño de los estudiantes en la solución de ejercicios matemáticos, a través de tareas para el desarrollo del pensamiento variacional en ámbitos fenomenológicos vinculados a la ingeniería.

El desarrollo del pensamiento variacional en el contexto ingenieril requiere de combinar la utilización del formalismo matemático con la comprensión de métodos matemáticos para cuantificar, describir y pronosticar cambios y con el desarrollo del pensamiento relacional, como hilos conductores para comprender y determinar razones de cambio de procesos continuos contextualizados a las características tecnológicas de la sociedad actual donde debe ejercer su profesión el ingeniero (Aguirre y Gil, 2015; Murcia y Henao, 2015), lo cual requiere, además, del conocimiento del contenido pedagógico para la enseñanza de la Matemática (Wilson, 2016). Además, se debe promover el uso didáctico de la modelación, comunicación y reflexión matemática y del lenguaje matemático para la solución de problemas sobre los conceptos de función, derivada e integral (Argote y Jiménez, 2014; Huertas y Castañeda, 2013), contextualizados en tareas propias de la ingeniería y reconocer en ellas la existencia de relaciones variacionales y los objetos matemáticos que subyacen (Arrieta y Díaz, 2015).

En el marco de estas exigencias y considerando el objetivo de la investigación, se asumen como referentes teóricos a la teoría de las representaciones semióticas de Duval (1999), la teoría acción, proceso, objeto, esquema de Dubinsky (1991) y el enfoque socioepistemológico de la Matemática de Cantoral (2013). De 
estos referentes se retoman los aspectos relacionados con el tratamiento y la conversión de los registros de representación semiótica, los procesos de coordinación reflexiva para la encapsulación y desencapsulación del proceso-objeto, la comprensión de los conceptos matemáticos a partir de su funcionalidad, y con la resignificación de los saberes matemáticos. En relación a lo referido por Duval (1999) se asume que los modelos mentales que tienen los estudiantes sobre los conocimientos matemáticos son exteriorizados a través de representaciones semióticas que pueden ser patrones numéricos, geométricos o simbólicos, reconociendo en ellos sus características esenciales.

Para el tratamiento y conversión de patrones conocidos a cualesquiera de sus registros de representación semiótica, los autores acuden al razonamiento inductivo - deductivo para favorecer el proceso de argumentación, utilizando y evaluando estrategias que permitan reconocer las características invariantes de fenómenos que representen situaciones de variación y cambio en diferentes registros de representación semiótica. La teoría acción, proceso, objeto, esquema de Dubinsky (1996), se utiliza para explicar el proceso de encapsulación proceso-objeto, como un proceso de abstracción reflexiva en la que las acciones sobre objetos matemáticos conocidos, son interiorizadas como procesos y encapsuladas como objetos mentales de pensamiento, aspecto que los autores consideran esencial para la concepción didáctica de las tareas propuestas.

Desde este punto de vista, fue posible explicar los procesos de encapsulación y desencapsulación de los conceptos como la coordinación reflexiva de la comprensión de los procesos de tratamiento y conversión, de los diferentes registros de representación semiótica de los procesos de variación y cambio, como objetos del Cálculo Diferencial, con el proceso inverso, de la comprensión de dichos objetos como los procesos de tratamiento y conversión mencionados anteriormente. La comprensión de los conceptos matemáticos a partir de su funcionalidad es asumida desde el enfoque socioepistemológico de la Matemática de Cantoral (2013), y está orientado a significar los conceptos mediante el uso de los conocimientos.

En este sentido, para garantizar la funcionalidad en el desarrollo del pensamiento variacional, se exalta que lo que está en juego son los procesos de variación y cambio en ámbitos fenomenológicos vinculados a la ingeniería, más que los conceptos del Cálculo Diferencial en sí mismo, de tal forma que la significación de los conceptos, no solo tomen sentido desde lo geométrico, numérico, estocástico y métrico, sino por su funcionalidad en el contexto ingenieril, siendo este último el contexto de referencia de los conceptos estudiados. La resignificación de los saberes matemáticos, como categoría de análisis para el estudio socioepistemológico del saber (Cantoral, 2013), se asume con el objetivo de propiciar la significación de los objetos del Cálculo Diferencial, a partir de prácticas de referencias diversas como son los procesos de variación y cambio en ingeniería, para propiciar el enriquecimiento de los saberes construidos, con nuevos significados, revelando diferentes usos del conocimiento matemático, a partir del cambio de las prácticas de referencia.

\section{METODOLOGÍA PARA EL DISEÑO DE LA PROPUESTA DE TAREAS}

Se realizó una propuesta de tareas para intervenir la práctica escolar, con estudiantes que ya habían vencido el curso de Cálculo Diferencial de las carreras de ingeniería de la Universidad ISA de Santiago de los Caballeros, República Dominicana, su diseño estuvo conformado por tres etapas de trabajo:

Etapa A: Diagnóstico inicial del desempeño de los estudiantes en la solución de ejercicios matemáticos en los cuales deban emplear el pensamiento variacional.

Etapa B: Diseño de la propuesta de tareas para el desarrollo del pensamiento variacional. La propuesta de tareas está formada por: a) Tareas de gestión de la información a través de la comprensión de patrones variacionales (orientadas al trabajo conceptual); b) Tareas de desarrollo procedimental a través de las representaciones semióticas de los procesos de variación y cambio (orientadas al trabajo procedimental); $c$ ) Tareas de desarrollo conceptual de los procesos de variación y cambio en los contextos: geométricos, numéricos, estocásticos y métricos (orientadas al trabajo conceptual); d) Tareas de resignificación de los saberes matemáticos para integrarlos y transferirlos en prácticas sociales en contextos determinados (orientadas al trabajo procedimental).

Etapa C: Diagnóstico final del desempeño de los estudiantes en la solución de ejercicios matemáticos en los cuales deban emplear el pensamiento variacional.

El objetivo de las etapas A y $C$ fue el de valorar el nivel de desempeño de los estudiantes en la solución de ejercicios matemáticos, en ámbitos fenomenológicos vinculados a la ingeniería poniendo en juego el pensamiento variacional, asumiendo esta valoración como una de las funciones de la dirección del proceso de enseñanza aprendizaje y que está estrechamente relacionada con la organización, planificación y 
ejecución de dicho proceso (Pérez, 2006). En la etapa A, los resultados fueron utilizados para atender las diferencias individuales de los estudiantes en el desarrollo de la etapa $\mathrm{B}$, y para coordinar sistémicamente la ejecución de las tareas previstas. La etapa $\mathrm{C}$ constituyó el estudio experimental para valorar si hubo mejoría con respecto a los resultados de la etapa $A$.

Para realizar el diagnóstico de las etapas A y C, se valoró la propuesta de Carlson, Jacobs, Coe, Larsen y Hsu (2003) y a partir de la misma, se aplicó un criterio de expertos que permitió precisar los niveles del desempeño de los estudiantes en la solución de ejercicios matemáticos en los cuales deben emplear el pensamiento variacional, como se puede apreciar en la tabla 1. La etapa B estuvo orientada a la caracterización de la propuesta de tareas, previo a un análisis didáctico de su diseño (Pochulu, Font, y Rodriguez, 2016), y una adecuada preparación del profesor de Matemática (Barboza y Zapata, 2013).

En relación a la preparación del profesor, se requirió, además de su preparación matemática, su capacidad de ponerse en el lugar de sus estudiantes, de valorar su actividad, de meditar sobre las manifestaciones conductuales y cognitivas de estos, comprenderlos en toda su diversidad, y lo más importante, poder utilizar esa información para propiciar el desarrollo de sus potencialidades (Abreu, Naranjo, Rhea y Gallegos, 2016). Por cada grupo de tareas se precisaron las características generales, se especificó su rol en la búsqueda del nexo conceptual-procedimental, su objetivo y los elementos que deben desarrollarse a través de ellas (Báez, Pérez y Mola, 2015).

Las tareas de gestión de la información a través de la comprensión de patrones variacionales, estuvieron orientadas a la búsqueda de nociones conceptuales y su función fue lograr que los estudiantes reconocieran las estructuras invariantes contenidas en los procesos de variación y cambio. Para ello se requirió potenciar y movilizar los saberes internos de los estudiantes, lo que incluye variables de tipo cognitivo, afectivoemocionales, necesarias para lograr la capacidad de reconocimiento a partir de identificar sus debilidades y fortalezas, reconocer los recursos que posee a partir del vínculo afectivo y del papel que desempeñan las vivencias en el funcionamiento de la motivación para reconocer. El desarrollo didáctico de estas tareas requirió interrelacionar los siguientes aspectos: a) Orientación motivacional reflexiva en la adquisición de datos para el reconocimiento de los fenómenos de patrones en procesos físicos y/ o abstracto; b) Identificación de características para la clasificación en patrones de variación y cambio de procesos físicos y / o abstractos; y c) Transformación metacognitiva del patrón en uno de los registros de representación semiótica de los procesos de variación y cambio.

Las tareas de desarrollo procedimental a través de las representaciones semióticas, de los procesos de variación y cambio, estuvieron caracterizadas por la orientación inductiva y deductiva de la manipulación de las representaciones semióticas de dichos procesos, con énfasis en la conversión y el tratamiento de las representaciones semióticas y en la coordinación reflexiva para la encapsulación y desencapsulación de los conceptos. Su función fue lograr que los estudiantes pudieran hacer argumentaciones reflexivas de dichas representaciones, y su desarrollo estuvo condicionado por la interrelación didáctica de los siguientes elementos: a) Orientación inductiva en las acciones de conversión de representación semiótica de los procesos de variación y cambio; b) Orientación deductiva en la justificación de los procesos de variación y cambio a partir de sus representaciones semióticas; y c) Coordinación reflexiva para la encapsulación y desencapsulación de los procesos de variación y cambio.

El tercer grupo de tareas estuvo relacionado con el desarrollo conceptual de los procesos de variación y cambio en los contextos geométricos, numéricos, estocásticos y métricos, donde el tratamiento conceptual, de las tareas, fue lo que predominó. Estas se caracterizaron por la orientación ontológica en la funcionalidad y la significación de dicha funcionalidad en estos contextos. Su función fue propiciar la comprensión de los conceptos de variación y cambio a partir de su funcionalidad en los contextos anteriormente mencionados, teniendo en cuenta las siguientes características: a)Orientación ontológica en la funcionalidad de la variación y el cambio en los contextos geométricos, numéricos, estocásticos y métricos; b) Representación de la variación y el cambio como forma de expresión de su funcionalidad; y c) Significación de los procesos de variación y cambio desde su funcionalidad y su relación con otros objetos matemáticos.

En las tareas de resignificación de los saberes matemáticos, para integrarlos y transferirlos en prácticas sociales en contextos determinados, predominó lo procedimental y estuvieron orientadas a la reconstrucción reflexiva de significados, la explicitación de los procedimientos y argumentaciones que intervienen en las prácticas sociales para, por último, propiciar la movilización reflexiva de los saberes matemáticos para la toma de decisiones en la solución de problemas. Por eso el objetivo de estas tareas fue el adecuado desempeño de los estudiantes en la solución de problemas que involucren los conceptos de variación y cambio, a través del desarrollo de los siguientes elementos: a) Reconstrucción reflexiva de significados variacionales desde lo individual y lo social (institucional); b) Explicitación de los procedimientos, procesos- 
objetos y/o argumentaciones que intervienen en las prácticas sociales; y c) Movilización reflexiva, individual y social de los saberes matemáticos para favorecer la toma de decisiones.

En cada uno de los grupos de tareas se trabajó con actividades de predicción, transformación, aproximación, graficación-modelación, acumulación, estado permanente, analiticidad y variación, dirigidas a la construcción de nociones matemáticas en ámbitos fenomenológicos vinculados a la ingeniería (Moreno, Font y Juan, 2016; Arrieta Vera y Díaz Moreno, 2015; Camacho-Ríos, 2011; Cantoral, 2013), por ejemplo: la evolución de la levadura, las diluciones cerradas, la mecánica de fluidos, las gráficas por computadoras y la propagación del calor, entre otras.

Tabla 1: Niveles del desempeño de los estudiantes en relación al desarrollo del pensamiento variacional.

\begin{tabular}{|c|c|c|}
\hline Nivel & Características & Criterios valorativos \\
\hline $\begin{array}{l}\text { Reconocimiento } \\
\text { (Nivel 1) }\end{array}$ & $\begin{array}{l}\text { Como la expresión del nivel primario } \\
\text { en el desarrollo del pensamiento } \\
\text { variacional, donde se examinan de } \\
\text { forma global la existencia y el } \\
\text { significado de la variación en un } \\
\text { fenómeno modelado matemáticamente } \\
\text { y se distinguen los elementos en la } \\
\text { variación. }\end{array}$ & $\begin{array}{l}\text { Los estudiantes identifican y manipulan relaciones, } \\
\text { patrones, transformaciones, procesos algebraicos y } \\
\text { geométricos. } \\
\text { Los estudiantes reconocen la existencia y el significado } \\
\text { de la variación en un fenómeno modelado } \\
\text { matemáticamente. } \\
\text { Los estudiantes distinguen los elementos de la variación. }\end{array}$ \\
\hline $\begin{array}{l}\text { Relación } \\
\text { (Nivel 2) }\end{array}$ & $\begin{array}{l}\text { Es la expresión del nivel intermedio en } \\
\text { el desarrollo del pensamiento } \\
\text { variacional, donde dada una situación } \\
\text { variacional, representada por un } \\
\text { fenómeno modelado } \\
\text { matemáticamente, y por los elementos } \\
\text { que distinguen a la variación, se } \\
\text { puedan establecer las relaciones entre } \\
\text { dichos elementos expuestos en tablas, } \\
\text { gráficas y otras formas de } \\
\text { representación }\end{array}$ & $\begin{array}{l}\text { Los estudiantes establecen relaciones entre los } \\
\text { diferentes elementos que distinguen la variación, } \\
\text { relacionan propiedades y reglas siguiendo argumentos } \\
\text { deductivos informales. } \\
\text { Los estudiantes comunican y reflexionan } \\
\text { matemáticamente y usan el lenguaje matemático para } \\
\text { argumentar la solución de problemas. } \\
\text { Los estudiantes manipulan relaciones, transformaciones, } \\
\text { procesos algebraicos y geométricos. }\end{array}$ \\
\hline $\begin{array}{l}\text { Deducción formal } \\
\text { (Nivel 3) }\end{array}$ & $\begin{array}{l}\text { Es el más alto indicio de desarrollo del } \\
\text { pensamiento variacional en el } \\
\text { estudiante, el desempeño se concreta } \\
\text { en la solución de actividades que } \\
\text { modelan fenómenos ingenieriles. }\end{array}$ & $\begin{array}{l}\text { Los estudiantes descubren nuevas relaciones y realizan } \\
\text { clasificaciones inclusivas. } \\
\text { Los estudiantes comunican y reflexionan } \\
\text { matemáticamente y usan el lenguaje matemático para } \\
\text { argumentar la solución de problemas. } \\
\text { Los estudiantes comprenden las relaciones presentes en } \\
\text { fenómenos ingenieriles y reconocen en ellos la } \\
\text { existencia de relaciones variacionales y los objetos } \\
\text { matemáticos que subyacen en dichas situaciones. } \\
\text { Los estudiantes construyen nociones matemáticas en } \\
\text { ámbitos fenomenológicos vinculados a la ingeniería, a } \\
\text { través del estudio de prácticas sociales que hacen } \\
\text { emerger el conocimiento matemático. }\end{array}$ \\
\hline
\end{tabular}

\section{EJEMPLOS DE TAREAS PROPUESTAS}

Debido al poco espacio disponible, se presenta una de las actividades desarrolladas en la etapa C (Etapa experimental), para el proceso de enseñanza aprendizaje del concepto de derivada de una función real de una variable. En esta actividad se transitó por los cuatro tipos de tareas propuestas, en correspondencia con los tres niveles de desempeño, y sus correspondientes criterios valorativos, expuestos en la tabla 1.

Inicialmente se propuso el siguiente problema para examinar, de forma global, la existencia y el significado de la variación en un fenómeno modelado matemáticamente, así como para distinguir los elementos en la variación (Nivel 1, reconocimiento). 
Dos cosechadoras de arroz, en San Pedro de Macorís, comienzan su actividad saliendo desde el mismo punto de partida, una va de norte a sur a $7 \mathrm{~km} / \mathrm{h}$ y la otra de este a oeste a $5 \mathrm{~km} / \mathrm{h}$. Con el objetivo de estudiar el rendimiento de ambas cosechadoras, los especialistas que atienden la actividad agropecuaria se plantearon las siguientes interrogantes: Si ambas cosechadoras se desplazan sin detenerse, cuando la primera ha recorrido un $\mathrm{km}$, ¿cuán rápido se incrementa la distancia entre ellas?

A partir de este problema, la primera tarea se orientó a la gestión de la información, a través de la comprensión de patrones variacionales, la cual estuvo guiada a través de las siguientes preguntas: ¿Cuál es la variable independiente en el problema?; ¿Cuál es la dependencia de variables qué el problema revela?; ¿Existen otras variables dependientes en el problema?; ¿Cuáles son las relaciones entre las variables?; ¿Cómo se manifiestan las relaciones de variación y cambio entre las variables del problema? y ¿Qué se necesita encontrar para resolver el problema?

La segunda tarea se orientó al desarrollo procedimental, a través de las representaciones semióticas, lo que conllevó a la materialización semiótica del problema dado inicialmente, a través del proceso de conversión del registro verbal al registro gráfico (Figura 1).

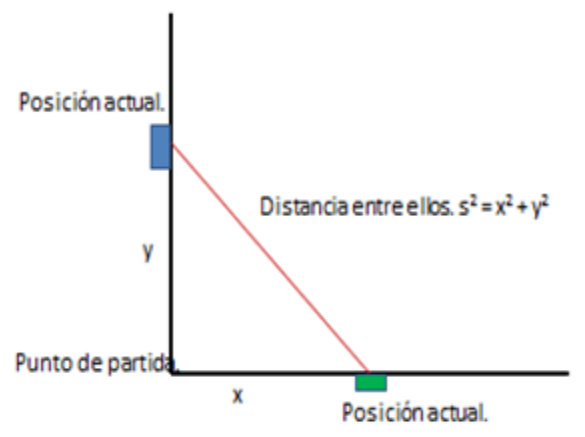

Fig. 1: Materialización semiótica del problema

Esta tarea se caracterizó por una orientación didáctica que propició el razonamiento inductivo - deductivo de lo procedimental, para que el estudiante ejecutara y argumentara los procedimientos de conversión del problema dado, en diversos registros de representación semiótica.

Posteriormente, la actividad se orientó al establecimiento de las relaciones entre los elementos expuestos en la gráfica, y en la ecuación encontrada y por los elementos que distinguen a la variación y el cambio (Nivel 2, relación), por lo que se realizó la tercera tarea orientada al desarrollo conceptual de los procesos de variación y cambio en el contexto algebraico y geométrico, propiciando la significación del concepto de derivada, no sólo desde lo geométrico y lo algebraico; sino, a través de la comprensión de los procesos de variación y cambio, a partir de su funcionalidad en el problema dado.

Para el desarrollo de esta tarea se trabajó en equipo, y se realizó un taller, donde se reflexionó y debatió sobre las relaciones entre los diferentes elementos que distinguen la variación, se discutieron propiedades y reglas, específicas del concepto derivada, siguiendo argumentos deductivos informales y exigiendo el uso del lenguaje matemático para argumentar la solución del problema.

Por último, la actividad se orientó a la solución de un nuevo problema, en el que se modelan fenómenos ingenieriles (Nivel 3, deducción formal), por lo que se desarrolló la tarea de resignificación de los saberes matemáticos, para integrarlos y transferirlos en prácticas sociales en contextos determinados.

Para el desarrollo de dicha tarea se remitió a los estudiantes al diagrama de flujo dado por Serna, Vergara y Flórez (2011), el cual describe las características físicas del segmento "Adecuación de Leche"., donde se muestra el camino de transformaciones físicas y químicas a las cuales se ve sometida la leche pasteurizada en este proceso de adecuación (Figura 2).

Se orientó a los estudiantes revisar el artículo donde aparece el diagrama de flujo, para que comprendieran su representación en la Ingeniería Química, destacándose que las líneas negras representan el flujo de leche líquida pasteurizada, y se les planteó la necesidad de hacer otra salida del tanque de almacenamiento para destinarlas a otros procesos de producción de productos lácteos, teniendo el mismo punto de partida y en posición perpendicular, a la salida existente. Los especialistas necesitan estudiar la eficiencia de los procesos que ocurren en ambas salidas, por lo que es necesario utilizar un instrumento que mida la velocidad del fluido de la leche. Se desprecia en el análisis de esta situación las pérdidas que pueden ocurrir en las tuberías a causa de la fricción. 


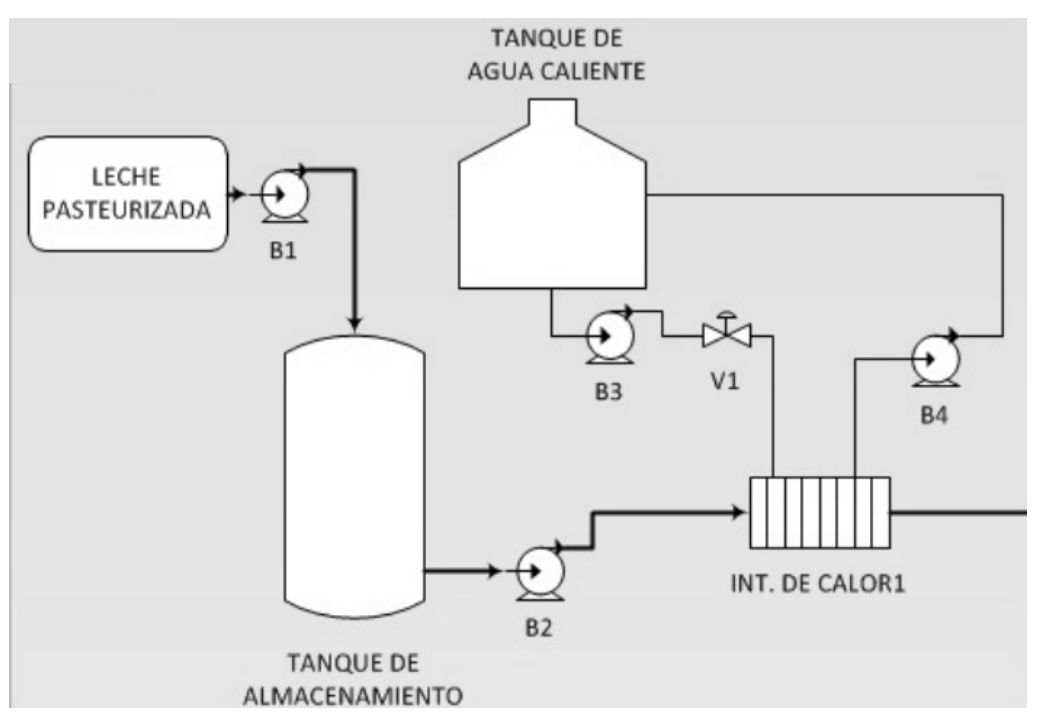

Fig. 2: Diagrama de flujo de segmento de proceso Adecuación de Leche, tomada de Serna, Vergara y Flórez (2011)

Ante este proyecto se solicitó a los estudiantes, trabajar en equipo, propiciando que reflexionaran matemáticamente, y utilizaran el lenguaje matemático, para argumentar la solución del problema, analizando la existencia de las relaciones variacionales, y de los objetos matemáticos que subyacen en la situación analizada, con la intención de dar significado al concepto de derivada, mediante el uso de sus conocimientos en contextos tecnológicos de producción de leche.

\section{METODOLOGÍA DEL ESTUDIO EXPERIMENTAL}

El estudio fue experimental y prospectivo, y se consideró como punto de partida la siguiente hipótesis: "Si se implementa una propuesta didáctica, que tenga en cuenta los niveles del desempeño y la realización de cuatro tipos de tareas para el desarrollo del pensamiento variacional, entones mejorará el desempeño de los estudiantes en la solución de ejercicios matemáticos donde tengan que emplear el pensamiento variacional en ámbitos fenomenológicos vinculados a la ingeniería". Se realizó al finalizar el segundo semestre del año 2015 en la Universidad ISA de Santiago de los Caballeros, República Dominicana. El universo estuvo constituido por los estudiantes que recibieron la docencia de Cálculo Diferencial, pero que aún no habían realizado el examen final. Tuvo una duración de 20 días y constituyó un espacio para, además de realizar el estudio, preparar a los estudiantes para el examen final de esta materia.

La muestra fue de tipo no probabilística y estuvo compuesta por 65 estudiantes que cumplieron los siguientes criterios de inclusión, que también se consideran de exclusión: a).Estudiantes que recibieron la materia Cálculo Diferencial y que se presentarían a examen; y b).Estudiantes que aceptaran participar en la investigación mediante la firma de un documento denominado consentimiento informado, donde se les haga saber el objetivo de esta. Como instrumento para determinar los niveles de desempeño en que se encontraban los estudiantes, se desarrolló una actividad similar a la descrita en el apartado anterior, para propiciar el tránsito por los tres niveles de desempeño (reconocimiento, relación y deducción formal), los cuales fueron valorados cualitativamente por la profesora, teniendo en cuenta los criterios valorativos expuestos en la tabla 1.

El trabajo directo con los estudiantes fue realizado por una profesora de Matemática de la Universidad ISA de Santiago de los Caballeros, República Dominicana, asesorada por un equipo de expertos de Cuba. Para el análisis estadístico se utilizó el lenguaje $R$, el cual es considerado como un entorno y lenguaje de programación, con enfoque al análisis estadístico. Constituye una implementación de software libre del lenguaje $\mathrm{S}$ pero con soporte de alcance estático. Se trata de uno de los lenguajes más utilizados en investigación por la comunidad estadística, muy popular en el campo de la minería de datos, la investigación biomédica, la bioinformática y la matemática financiera (Kleiber y Zeileis, 2008).

R es una herramienta sumamente útil ya que además de ser un programa de código abierto y gratuito tiene capacidad de análisis y poder de cálculo estadístico, provee operaciones estadísticas y brinda un lenguaje de programación que puede ser usado para crear gráficos, nuevas funciones o extender las actuales, y ofrece la posibilidad de trabajar desde estadísticas simples hasta tópicos más avanzados como análisis multivariado, modelos complejos de estructura de covariancia, entre otros. 
Se utilizaron tres técnicas estadísticas: el test de Shapiro Wilks, el test de Independencia para dos variables y el test de Wilcoxon. El Test de Shapiro-Wilk tradicionalmente es utilizado para contrastar la normalidad de un conjunto de datos y plantea como hipótesis nula que una muestra $x_{1}, x_{n}$ proviene de una población normalmente distribuida. Es considerado uno de los test más potentes para el contraste de normalidad, sobre todo para muestras pequeñas $(n<100)$. Para el test de Independencia para dos variables se utilizó el coeficiente de correlación de Pearson, el cual constituye una medida de la relación lineal entre dos variables aleatorias cuantitativas y es utilizado como un índice que puede utilizarse para medir el grado de relación de dos variables siempre y cuando ambas sean cuantitativas (Benesty, Chen, Huang y Cohen, 2009). Este test se utilizó para analizar la independencia de las variables teniendo en cuenta el coeficiente de correlación de Pearson, donde $\mathrm{H}_{0}$ plantea que las muestras son independientes y la hipótesis alternativa que estas son pareadas, teniendo en cuenta su grado de relación. Por último, la prueba de los rangos con signo de Wilcoxon es una prueba no paramétrica para comparar la mediana de dos muestras relacionadas y determinar si existen diferencias entre ellas. Se utilizó como alternativa a la prueba t de Student para cuando no se puede suponer la normalidad de dichas muestras (Wilcoxon, 1945).

\section{RESULTADOS Y DISCUSIÓN}

Para validar las tareas implementadas se compararon los dos grupos de 65 estudiantes, divididos en grupo de control y experimental. La metodología seguida en el estudio comparativo tuvo como referente al estudio comparativo realizado por Valerio et al, (2016), quien de manera similar utilizan grupos de control y experimental para validar sus resultados. Se asumió el grupo de control como un elemento esencial del experimento científico controlado, para evitar que las apariencias condujeran a conclusiones erróneas y la propuesta de tarea se aplicó solamente en el grupo experimental. Se compararon lo resultados obtenidos y se consideró que si la proporción de resultados deseados fuese mayor en el grupo experimental que en el grupo de control, entonces la propuesta de tareas se consideraría eficiente para el desarrollo del pensamiento variacional, si fuese igual, ineficiente, y si es menor, contraproducente.

Con los resultados del diagnóstico final, se analizó la proporción de los resultados deseados, teniendo en cuenta los niveles del desempeño de los estudiantes en relación al desarrollo del pensamiento variacional. Con este experimento se quiso analizar si las tareas propuestas fueron eficientes o no, en dependencia de la proporción de los estudiantes del nivel de relación y de deducción formal (nivel 2 y 3) con respecto al nivel de reconocimiento (nivel 1) del grupo experimental en relación al grupo de control. En la Tabla 2, se muestra la cantidad de estudiantes por niveles, tanto del grupo experimental como el de control. Como se puede apreciar, la proporción del grupo experimental: (Cantidad de estudiantes en el nivel de Relación + Cantidad de estudiantes en el nivel de Deducción formal) / (Cantidad de estudiantes en el nivel de Reconocimiento) $=$ $(30+20) / 15=50 / 15 \approx 3.333$ supera al del grupo de control $(17+18) / 30=35 / 30 \approx 1.667$. Por lo que se puede concluir que la propuesta es eficiente.

Tabla 2: Cantidad de estudiantes por niveles de desempeño de los estudiantes.

\begin{tabular}{|c|c|c|c|}
\hline Grupo & $\begin{array}{c}\text { Cantidad de estudiantes en el } \\
\text { nivel de Reconocimiento }\end{array}$ & $\begin{array}{c}\text { Cantidad de estudiantes en } \\
\text { el nivel de Relación }\end{array}$ & $\begin{array}{c}\text { Cantidad de estudiantes en el } \\
\text { nivel de Deducción formal }\end{array}$ \\
\hline Experimental & 15 & 30 & 20 \\
\hline Control & 30 & 17 & 18 \\
\hline
\end{tabular}

\section{Comparación entre los resultados de los grupos de estudiantes}

Para analizar si existían diferencias significativas entre las calificaciones de los estudiantes que conformaron los dos grupos (experimental y de control), se realizó el análisis de las variables gExperimental (recoge las calificaciones de los estudiantes del grupo experimental) y gControl (recoge las calificaciones de los estudiantes del grupo de control), cuyos valores se encuentran entre 2 y 5 puntos.

Luego se implementó un script en lenguaje $\mathrm{R}$ para comparar las muestras de las calificaciones de los estudiantes del grupo experimental y de control, donde se analizó la normalidad de las variables gExperimental y gControl, con el objetivo de seleccionar un test de comparación de grupos apropiado: 
gExperimental<-

c $(4,4,3,4,4,4,5,5,3,5,5,5,4,4,4,3,5,5,4,3,4,5,3,4,3,5,4,5,3,5,3,4,4,5,4,3,5,5,4,4,3,5,5,3,5,4,4,5,3,4,4,4,4,3,4,4,4$ $, 5,3,5,4,4,3,4,4)$

\section{Análisis y Discusión de las Pruebas Estadísticas}

Como las muestras fueron menores que 100 elementos, cada una, se seleccionó el test de Shapiro-Wilk para analizar la normalidad de las variables, el cual plantea que las hipótesis nula y alternativa:

$\mathrm{H}_{0}$ : proviene de una población normalmente distribuida

$\mathrm{H}_{1}$ : no proviene de una población normalmente distribuida

>shapiro.test(gControl)

Shapiro-Wilk normality test

data: gControl

$W=0.66913, p$-value $=7.952 e-11$

>shapiro.test(gExperimental)

Shapiro-Wilk normality test

data: gExperimental

$W=0.80769, p$-value $=8.88 \mathrm{e}-08$

Al ser los valores de los $p$-value menores que 0.05 se rechaza la hipótesis nula $\left(\mathrm{H}_{0}\right)$ por lo que se puede decir que esta variable no tiene normalidad, o sea los datos de la variable no siguen una distribución normal. Luego se analizó la correlación entre los grupos, lo cual permitió hacer estimaciones del valor de una de ellas conociendo el valor de la otra variable. Los coeficientes de correlación son medidas que indican la situación relativa de los mismos sucesos respecto a las dos variables, es decir, son la expresión numérica que nos indica el grado de relación existente entre las 2 variables y en qué medida se relacionan. Son números que varían entre los límites +1 y -1 . Su magnitud indica el grado de asociación entre las variables; el valor $r=0$ indica que no existe relación entre las variables; los valores (1 son indicadores de una correlación perfecta positiva (al crecer o decrecer $\mathrm{X}$, crece o decrece $\mathrm{Y}$ ) o negativa (Al crecer o decrecer $\mathrm{X}$, decrece o crece $Y$ ).

En el script se ejecuta:

$\geq \operatorname{cor}(g$ Experimental, gControl)

[1] 0.2248107

Para interpretar el coeficiente de correlación se puede utilizar la escala mostrada en la Tabla 1.

Tabla 3: Valores del coeficiente de correlación

\begin{tabular}{|c|c|}
\hline Valor & Significado \\
\hline-1 & Correlación negativa grande y perfecta \\
\hline-0.9 a -0.99 & Correlación negativa muy alta \\
\hline-0.7 a -0.89 & Correlación negativa alta \\
\hline-0.4 a -0.69 & Correlación negativa moderada \\
\hline$-0,2$ a $-0,39$ & Correlación negativa baja \\
\hline-0.01 a -0.19 & Correlación negativa muy baja \\
\hline 0 & Correlación nula \\
\hline 0.01 a 0.19 & Correlación positiva muy baja \\
\hline 0.2 a 0.39 & Correlación positiva baja \\
\hline 0.4 a 0.69 & Correlación positiva moderada \\
\hline 0.7 a 0.89 & Correlación positiva muy alta \\
\hline 0.9 a 0.99 & Correlación positiva grande y perfecta \\
\hline 1 & \\
\hline
\end{tabular}


Como se pudo apreciar, el valor de la correlación está en el intervalo de 0.2 a 0.39 , lo cual significa que existe una correlación positiva baja entre los grupos, por lo tanto, los dos grupos son independientes. El siguiente comando del script permite graficar la correlación de los datos de los grupos.

\section{>plot(gExperimental gControl)}

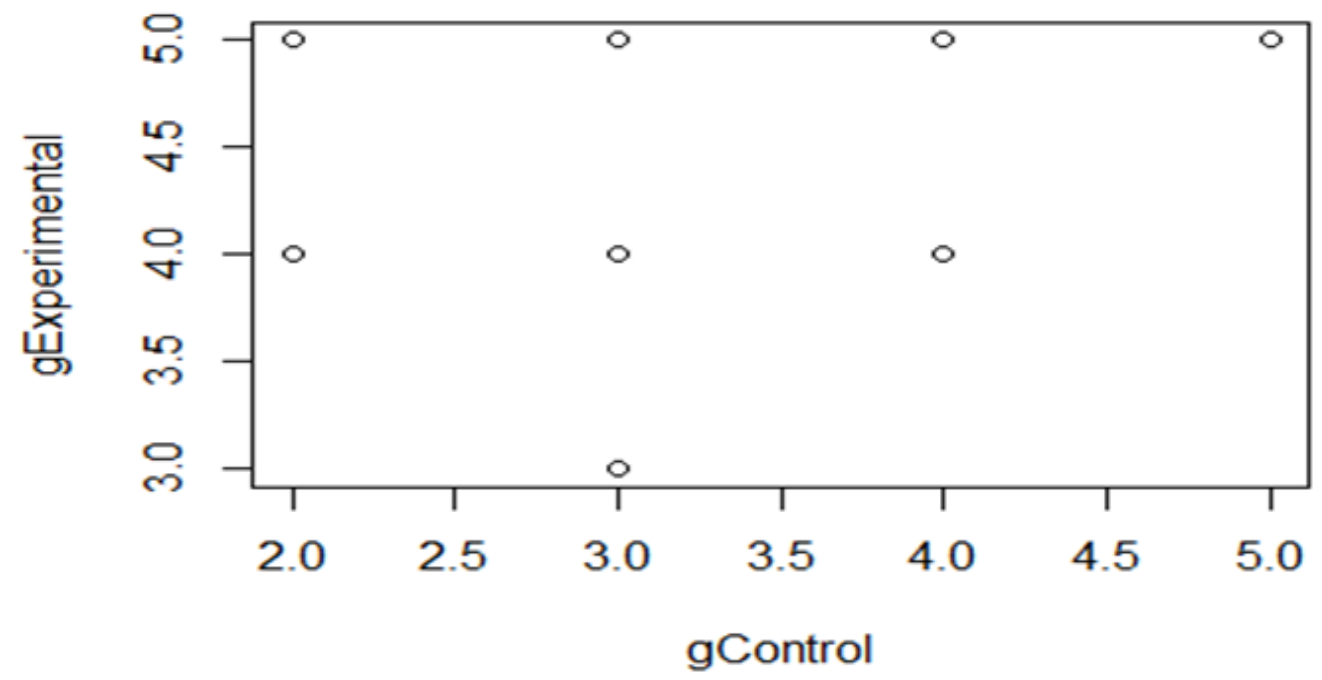

Fig. 3: Gráfico de dispersión de la correlación de los datos de las calificaciones de los estudiantes de los grupos de control y experimental.

Como los grupos no son paramétricos e independientes, se procedió a seleccionar una prueba de comparación de grupos que se ajuste a estas condiciones, en este caso, la prueba de $U$ de Mann Whitney para comparar 2 medianas, la cual se ejecuta en R:

\section{>wilcox.test(gExperimental, gControl)}

Wilcoxon rank sum test with continuity correction

data: gExperimental and $g$ Control

$W=3292.5, p$-value $=2.655 e-09$

alternative hypothesis: true location shift is not equal to 0

Para interpretar estos resultados, se debió decidir entre la hipótesis nula y alternativa a comparar:

1) $\quad H_{0}$ : Las 2 medias poblacionales son iguales

$H_{1}$ : Las 2 medias son diferentes

2) Fijar un nivel de probabilidad de equivocarse: $\alpha=0.05$

Si p-value $>\alpha=0.05$ entonces se acepta $H_{0}$, sino se rechaza $H_{0}$ y se acepta $H_{1}$.

Como el $p$-value fue menor que 0.05 se rechazó la hipótesis $\mathrm{H}_{0}$ referida a que las medias de las muestras son iguales, por lo que los grupos fueron diferentes. Teniendo en cuenta las medias, este resultado sugirió que al aplicar la propuesta de tareas hubo algún cambio en el aprendizaje de los estudiantes.

Luego de graficar la frecuencia de los valores de los niveles de los dos grupos se pudo concluir que existió notable mejoría en el desempeño de los estudiantes después de aplicar la etapa B de la propuesta, ya que aumentó la cantidad de estudiantes clasificados en los niveles de desempeño 2 y 3 (Figura 4).

Además, teniendo en cuenta el valor de la moda para los niveles del desempeño de los estudiantes en relación al desarrollo del pensamiento variacional de los grupos experimental y de control, cuyos valores para estas muestras son Nivel 2 y Nivel 3 respectivamente, se pudo concluir que su implementación favoreció el proceso de desarrollo del pensamiento variacional de los estudiantes en el Cálculo Diferencial. 


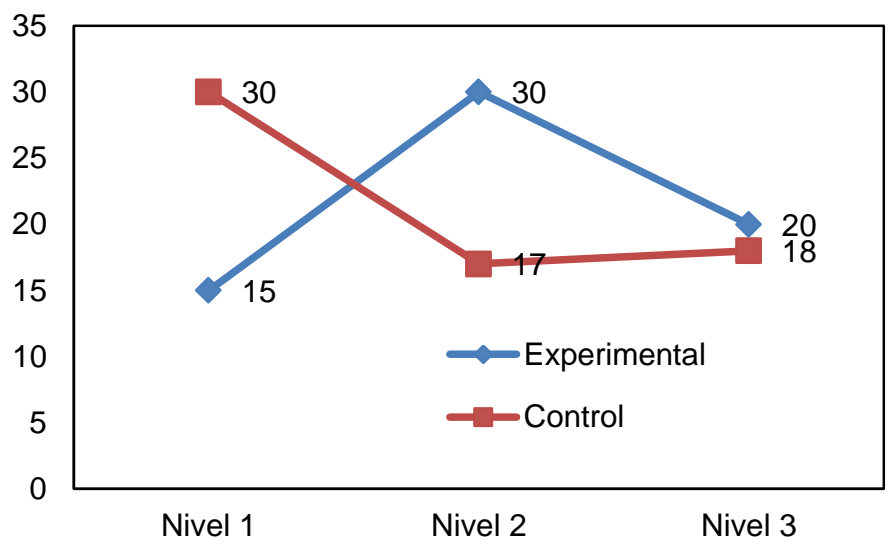

Fig. 4: Comportamiento de los niveles de desempeño de los estudiantes en los grupos experimental y de control

\section{CONCLUSIONES}

Los resultados expuestos, permitieron valorar el desempeño de los estudiantes, en la solución de ejercicios matemáticos donde se emplea el pensamiento variacional en ámbitos vinculados a la ingeniería. Se mostró evidencias de que la propuesta de las tareas influye eficientemente en el desarrollo del pensamiento matemático de los estudiantes, pues al enfocarlas desde los procesos de variación y cambio, se favorece el pensamiento variacional en contextos ingenieriles. Según García y Benítez (2013), existe una estrecha relación entre el razonamiento que los estudiantes realizan al trabajar en una tarea y la forma en que esta se presenta. En este sentido, los resultados obtenidos en esta investigación se convierten en una propuesta que puede ser aplicable en diferentes cursos de Ingeniería.

Lo importante de la propuesta de tareas es que se propicia la comprensión y caracterización, de las relaciones presentes en fenómenos ingenieriles y los saberes matemáticos que subyacen en el mismo, esto confirma la necesidad planteada por Cantoral (2013), de hacer un cambio de enfoque en el discurso matemático escolar de las materias de Cálculo Diferencial, dejando atrás la solución de problemas aplicados a la ingeniería como la etapa de sistematización de los contenidos, y el énfasis en estudiar los objetos matemáticos sin tener en cuenta su funcionalidad, para pasar al análisis de prácticas sociales que hacen emerger el conocimiento matemático, por tanto, lo que llevaría un cambio de objetos a prácticas.

\section{AGRADECIMIENTOS}

Agradecimiento al apoyo brindado a la Universidad ISA de Santiago de los Caballeros, República Dominicana y al Proyecto Nacional del Departamento de Matemática de la Universidad de Camagüey, "Perfeccionamiento de la enseñanza de la Matemática" (código PP221LH053), asociado al Programa Nacional Problemas Actuales del Sistema Educativo Cubano. Perspectivas de desarrollo, del Ministerio de Educación de Cuba.

\section{REFERENCIAS}

Abreu, O., Naranjo, M. E., Rhea, B.S., Gallegos, M.C., Modelo Didáctico para la Facultad de Ciencias Administrativas y Económicas de la Universidad Técnica del Norte en Ecuador, doi: 10.4067/S071850062016000400002, Formación Universitaria9(4), 3-10 (2016)

Aguirre, E. A., y Gil, S. G., Articulación de la robótica como eje de la formación interdisciplinar en el programa de Tecnología en Electrónica de la UNIMINUTO Sede Principal, Revista Inventum, 16,26-32 (2015)

Almeida, L, Palharini, B., The" Worlds of Mathematics" in Mathematical Modelling Activities, Bolema, 26(43), 907-934 (2012)

Argote, I. M., y Jiménez, R. A., Matemáticas para la Ingeniería de Sistemas, Ventana Informática, 30,157-173 (2014)

Arrieta Vera, J., y Díaz Moreno, L., Una perspectiva de la modelación desde la Socioepistemología, Revista latinoamericana de investigación en matemática educativa, 18(1), 19-48 (2015) 
Báez, A., Pérez, R., y Mola, C., Tratamiento didáctico de los procesos de variación y cambio y el nexo conceptual procedimental. Informe de investigación doctoral, Universidad de Camaguey, Cuba (2015)

Barboza, J., y Zapata, H. A., El Estudio de Clase, Estrategia y Escenario para la Cualificación del Profesor de Matemáticas. Form. Univ., 6(4), 49-62 (2013)

Benesty, J., Chen, J., Huang, Y., e Cohen, I., Pearson correlation coefficient. In Noise reduction in speech processing, Springer Berlin Heidelberg (2009)

Cabeza, C., Mendoza, M., Manifestaciones Emergentes del Pensamiento Variacional en Estudiantes de Cálculo Inicial, doi: 10.4067/S0718-50062016000600003, Formación Universitaria, 9(6), 13-26, (2016)

Camacho-Ríos, A., Socioepistemología y prácticas sociales, Hacia una enseñanza dinámica del cálculo diferencial, Revista Iberoamericana de Educación Superior, 2(3),152-171 (2011)

Cantoral, R., Teoría socioepistemológica de la Matemática Educativa, Estudios sobre construcción social del conocimiento ( $1^{\text {st }}$ Ed.), Editorial Gedisa S.A., Barcelona (2013)

Cantoral, R., y Farfán, R., Pensamiento y lenguaje variacional en la introducción al análisis, Épsilon, 42, 353369 (1998)

Cantoral, R., Desarrollo del pensamiento y lenguaje variacional, una mirada socioepistemológica, Acta Latinoamericana de Matemática Educativa, 17(1), 1-9 (2004)

Carlson, M., Jacobs, S., Coe, E., Larsen, S., y Hsu, E., Razonamiento covariacional aplicado a la modelación de eventos dinámicos: Un marco conceptual y un estudio, EMA, 8(2), 121-156 (2003)

Cuevas, C., Promoviendo el pensamiento funcional en la enseñanza del cálculo: un experimento con el uso de tecnologías digitales y suordos resultados, Annales De Didactique Et De Sciences Cognitives, 17, (137 - 168) (2012)

De Las Fuentes, M., Arcos, J. L. y Navarro, C. R., Impacto en las Competencias Matemáticas de los Estudiantes de Ecuaciones Diferenciales a Partir de una Estrategia Didáctica que Incorpora la Calculadora, doi: 10.4067/S0718-50062010000300005, Formación Universitaria, 3(3), 33-44 (2010)

Dubinsky, E., The constructive aspects of reflective abstraction in advanced mathematics: Epistemological Foundations of Mathematical Experiences, New York: Springer-Verlag (1991)

Duval, R., Semiosis y pensamiento humano: registros semióticos y aprendizajes intelectuales, $1^{\text {a }}$ Ed., traducción al español a cargo de M. Vega, 1-314, Universidad del Valle Grupo de Educación Matemática, Cali, Colombia (1999)

Font, V., Godino, J., y Gallardo, J., The emergence of objects from mathematical practices, Educational Studies in Mathematics, 82(1), 97-124 (2013)

García, M.L., Benítez, A.A., Diseño e Implementación de Tareas para Apoyar el Aprendizaje de las Matemáticas, doi: 10.4067/S0718-50062013000100003, Formación Universitaria, 6(1), 13-20,(2013)

Hannula, M., Space, time and number in the brain: searching for the foundations of mathematical thought, Research in Mathematics Education, 15(2), 205-208 (2013)

Huertas, J. C., y Castañeda, Y. M., Funciones en contexto, Una experiencia enriquecida en la modelación y simulación interactiva, Sistemas \& Telemática, 11(26),59-80,(2013)

Kleiber, C., y Zeileis, A., Applied econometrics with R, Springer Science \& Business Media (2008)

Kleiner, I., History of the infinitely small and the infinitely large in calculus, Educational Studies in Mathematics, 48(2-3), 137-174 (2001)

Moreno, N., Font, V., y Juan, R., La importancia de los diagramas en la resolución de problemas de cuerpos deformables en Mecánica: el caso de la fuerza de fricción, Revista chilena de ingeniería, 24(1), 158-172 (2016)

Murcia, M. E., y Henao, J. C., Educación matemática en Colombia, una perspectiva evolucionaria, Entre Ciencia e Ingeniería, 9(18),23-30 (2015) 
Ordoñez, S., y Alonso, E., Razones, proporciones y proporcionalidad en una situación de reparto: una mirada desde la teoría antropológica de lo didáctico, Revista latinoamericana de investigación en matemática educativa, 16(1), 65-97 (2013)

Paoloni, P. V., Martín, R. B., Chiecher, A. C.,Contextos de Aprendizaje en el Nivel Medio y en la Universidad. Percepciones y Expectativas de Estudiantes que Ingresan en Carreras de Ingeniería, doi: 10.4067/S071850062015000500007, Formación Universitaria, 8(6),47 (2015)

Pérez, O., ¿Cómo diseñar el sistema de evaluación del aprendizaje en la enseñanza de las matemáticas?, Revista latinoamericana de investigación en matemática educativa, 9(2), 267-297 (2006)

Pochulu, M., Font, V., \& Rodriguez, M., Desarrollo de la competencia en análisis didáctico de formadores de futuros profesores de matemática a través del diseño de tareas, Revista latinoamericana de investigación en matemática educativa, 19(1), 71-98 (2016)

Ruiz, E., Diseño de estrategias de enseñanza para el concepto de variación en áreas de ingeniería, Innovación Educativa, 9(46), 27-39 (2009)

Serna Quilindo, W. Y.,Vergara González, D. C., Flórez Marulanda, J. F., Procedimiento de modelado isa s88 para ejecución de órdenes de producción basadas en récipes, Ciencia e Ingeniería Neogranadina, 21(2),107130, Bogotá, ISSN: 0124-8170 (2011)

Valerio, G., Jaramillo, J., Caraza, R., Rodríguez, R., Principios de Neurociencia aplicados en la Educación Universitaria, doi: 10.4067/S0718-50062016000400009, Formación Universitaria 9(4), 75-82 (2016)

Wilcoxon, F., Individual comparisons by ranking methods, Biometrics bulletin, 1(6), 80-83 (1945)

Wilson, A., Knowledge for Equitable Mathematics Teaching: The Case of Latino ELLs in U.S. Schools, The Mathematics Enthusiast, 13(1), 111-129 (2016) 
